\title{
Primary and Solvent Kinetic Isotope Effects in the Water-Assisted Tautomerization of Formamidine: An ab Initio Direct Dynamics Study
}

\author{
Robert L. Bell and Thanh N. Truong* \\ Department of Chemistry, University of Utah, Salt Lake City, Utah 84112
}

Received: June 3, 1997; In Final Form: July 23, $1997^{\otimes}$

\begin{abstract}
We present an ab initio direct dynamics study of the primary and solvent kinetic isotopes effects (KIEs) for the water-assisted tautomerization in the formamidine-water complex. These calculations are based on a variational transition state theory plus multidimensional semiclassical tunneling corrections with potential energy information calculated at the MP2 level of theory using the 6-31G(d,p) basis set. We found that both the primary and solvent KIEs are large at low temperatures and are due not only to tunneling but also to quantum effects in vibrational motions. The primary KIEs were larger than the solvent KIEs. This results from differences due to the effect of deuteration on vibrational modes. Bending modes show significant inverse KIEs, while those of the reactive $\mathrm{OH}$ and $\mathrm{NH}$ vibrations show both inverse and normal effects. Such effects can be explained by examining changes in the zero-point energies of these modes. The adherence of the $\mathrm{HH} / \mathrm{HD} / \mathrm{DD}$ rates to the rule of the geometric mean is also examined.
\end{abstract}

\section{Introduction}

For decades, studies of kinetic isotope effects (KIEs), whether primary, secondary, or solvent, have been carried out to aid in the determination of chemical and biochemical pathways and reaction mechanisms. ${ }^{1-21}$ Since many reactions of chemical and biochemical interest occur in aqueous solution, the study of water-assisted proton-transfer reactions is of great importance. The study of KIEs in such reactions is of particular interest. There have been numerous experimental ${ }^{1-14,22,23}$ and theoretical ${ }^{15-21,24-34}$ studies performed on proton transfer in systems of chemical and biochemical interest.

While recognizing the importance of experimentally determined KIEs, theoretical studies can examine further details of chemical processes that are not easily accessible to experimental determination. For instance, within the transition state theory formalism, one can factor the rate constant into contributions of individual motions, i.e., translation, rotation, vibration, and tunneling. An examination of the effect of these individual contributions on the KIE will give detailed information on the mechanism of a particular chemical process. ${ }^{28,32}$

Another aspect of KIEs involves the rule of the geometric mean (RGM), which has been used to help determine the mechanisms (i.e. whether concerted or stepwise) of various double proton-transfer reactions..$^{1,3-5,9-11}$ This rule states that isotopic disproportionation equilibrium constants are nearly identical with the classical value. ${ }^{35}$ It was proven to be exact for systems obeying partition functions with small quantum corrections. ${ }^{35}$ As a consequence, isotopic rate constants for concerted double proton-transfer reactions are related by $k_{\mathrm{HH}} /$ $k_{\mathrm{HD}}=k_{\mathrm{HD}} / k_{\mathrm{DD}}$, which resulted from combining equilibrium isotope effects and transition state theory. ${ }^{35-37}$ Double protontransfer reactions deviating from this rule mostly have been interpreted as being either the result of tunneling ${ }^{9-11,38}$ or the result of a stepwise process via an intermediate..$^{5,9-11,38,39}$ The validity of the RGM has not yet been investigated theoretically on a double proton-transfer reaction where tunneling is significant. Such a study would be of fundamental interest for further understanding mechanisms of double proton-transfer reactions.

${ }^{\otimes}$ Abstract published in Advance ACS Abstracts, September 15, 1997.
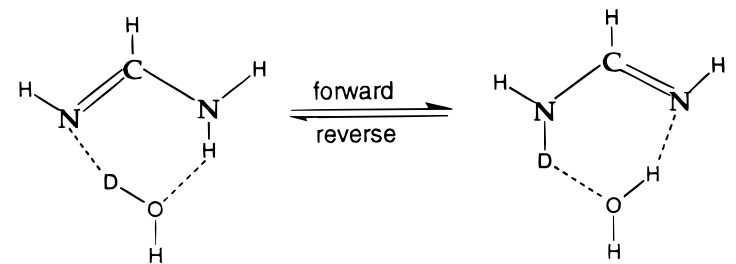

Figure 1. Water-assisted tautomerization in the formamidine-water complex. The forward direction is used to model the solvent KIE, and the reverse direction is used to model the primary KIE.

In this study, we present an ab initio direct dynamics investigation of the primary and solvent deuterium KIE on the water-assisted tautomerization in the formamidine-water complex for the forward and reverse reactions as shown in Figure 1. The forward reaction can be viewed as a model for the solvent KIE and the reverse as a model for the primary KIE. As mentioned in our previous study, ${ }^{40}$ in addition to its own biological importance, this system can be used as a model system for proton-transfer reactions in DNA bases. ${ }^{41}$ This tautomerization can also be viewed as a simple model for the study of solvent kinetic isotope effects ${ }^{23,28,42}$ in the case where water solvent molecules participate directly in the proton-transfer process. This can be viewed as a first step toward modeling solvent KIEs in solution. A more complete solvation model would have to include the effect of hydrogen bonding from the first solvation shell and longer range solvent-solute interactions. The former may be accounted for through the specific inclusion of additional waters in the supermolecule approach and the latter by using a dielectric continuum model.

In our previous study on this system, ${ }^{40}$ we calculated thermal rate constants of the gas phase and water-assisted tautomerizations in formamidine using a canonical variational transition state theory (CVT) with multidimensional semiclassical tunneling corrections. We found three important results, namely: (i) the addition of a water to the gas phase molecule significantly stabilized the transition state, thus lowering the classical barrier to tautomerization by $26.9 \mathrm{kcal} / \mathrm{mol}$, (ii) tunneling is significant at low temperatures; even at $300 \mathrm{~K}$ it enhances the rate by 3 orders of magnitude for the water-assisted tautomerization of 
formamidine, and (iii) vibrational excitation of the solvent (water) symmetric stretch enhanced the rate of this tautmerization.

In this study, we continue our investigation of the dynamics and mechanics of this proton-transfer reaction. In particular, our goals are to examine (i) the temperature dependence of the primary and solvent KIEs and (ii) the adherence of the KIE to the rule of the geometric mean. ${ }^{35}$ This paper is organized as follows. Section II gives a brief outline of variational transition state theory and semiclassical multidimensional tunneling. Section III outlines the computational details. Section IV gives an analysis of the kinetic isotope effects and the rule of the geometric mean. Section $\mathrm{V}$ gives the results and discussion, and section VI provides the conclusion.

\section{Theory}

The canonical variational transition state theory (CVT) rate constant is determined by minimizing the generalized transition state (GTS) rate constant, $k^{\mathrm{GT}}(T, s)$, with respect to the location of the dividing surface that is perpendicular and intersects the reaction coordinate at $\mathrm{s}$. The reaction coordinate, $s$, is defined as the distance along the minimum energy path (MEP) with the origin at the transition state. Negative and positive values of $s$ correspond to reactant and product sides of the reaction path, respectively. The CVT rate constant is thus given by

$$
k_{\mathrm{CVT}}(T)=\min _{s} k_{\mathrm{GT}}(T, s)=k^{\mathrm{GT}}\left(T, s_{*}{ }^{\mathrm{CVT}}\right)
$$

where $s_{*}$ CVT is the CVT temperature-dependent dynamical bottleneck, i.e., the location of $s$ that minimizes the GTS rate constant. The GTS rate constant is given by

$$
k^{\mathrm{GTS}}(T, s)=\sigma \frac{k_{\mathrm{B}} T}{h} \frac{Q^{\mathrm{GTS}}(T, s)}{Q^{\mathrm{R}}(T)} \mathrm{e}^{-V_{\mathrm{MEP}}(s) / k_{\mathrm{B}} T}
$$

where $\sigma$ is the symmetry factor and has a value of 1 for both the forward and reverse reactions, $k_{\mathrm{B}}$ is Boltzmann's constant, $h$ is Planck's constant, $V_{\mathrm{MEP}}(s)$ is the Born-Oppenheimer potential along the MEP that is the steepest descent path from the transition state toward the reactant and product, $Q^{\mathrm{GTS}}(T, s)$ is the internal partition function at the generalized transition state at $s$, and $Q^{\mathrm{R}}(T)$ is the reactant partition function. The total internal quantum partition functions can be written as products of the individual quantum partition functions $Q_{\text {elec}}, Q_{\text {rot }}$, and $Q_{\text {vib }}$, by assuming that the electronic, rotational, and vibrational degrees of freedom are separable. Due to small energy level spacing, rotational partition functions can be treated classically. The electronic partition functions for both the generalized transition state and reactant are assumed to cancel. Vibrational partition functions were calculated quantum mechanically within the harmonic independent normal mode approximation (INM) and can be written as a product of $3 N-7$ GTS individual vibrational partition functions for nonlinear molecules, $q_{\mathrm{vib}, i}$, as

$$
Q_{\mathrm{vib}}=\prod_{i=1}^{3 N-7} q_{\mathrm{vib}, j}
$$

where $N$ is the number of atoms in the system. Note that in the calculation of the CVT rate constant, motion along the reaction coordinate is still treated classically.

Quantum mechanical effects, i.e., tunneling, along the reaction coordinate are included by multiplying the CVT rate constant by the ground-state transmission coefficient, $\kappa^{\mathrm{CVT} / \mathrm{G}}$, to give

$$
k^{\mathrm{CVT} / \mathrm{G}}(T)=\kappa^{\mathrm{CVT} / \mathrm{G}}(T) k^{\mathrm{CVT}}(T)
$$

There exists several approximations to account for tunneling effects along the reaction coordinate. Only two will be used in this study, namely, the one-dimensional Wigner (W) and the multidimensional semiclassical small-curvature tunneling (SCT) approximations, which are the most accurate and feasible methods within our ab initio direct dynamics approach.

The SCT approximation is a generalization of the MarcusColtrin approximation, ${ }^{43}$ which assumes that the tunneling path is distorted from the MEP out to a concave-side vibrational turning point in the direction of the internal centrifugal force. The path is not actually calculated, but instead the centrifugal effect is included in the reduced mass, yielding an effective reduced mass. It is called the centrifugal-dominant smallcurvature semiclassical adiabatic ground-state (CD-SCSAG) approximation, which is abreviated SCT (small-curvature tunneling). Both the Wigner ${ }^{4}$ and the SCT approximations are discussed in detail elsewhere. ${ }^{45-47}$

\section{Computational Details}

The minimum energy path for the forward and reverse tautomerization in the formamidine-water complex was calculated using the second-order Gonzalez and Schlege ${ }^{48}$ method in mass-weighted Cartesian coordinates with a step size of 0.04 $\mathrm{amu}^{1 / 2} \mathrm{a}_{0}$ for a total of 50 points on each side of the transition state. This was done for the protonated, monodeuterated, and double-deuterated systems. Note that since the MEP is symmetric for the protonated and double-deuterated systems, only half of the MEP was calculated. However, for the monodeuterated system, the MEP is not symmetric and therefore both sides of the MEP were calculated. Hessian calculations were performed at 40 selected points along the MEP according to the focusing technique. ${ }^{49}$ All geometry optimizations, MEP, and frequency calculations were done at the MP2/6-31G(d,p) level of theory using the $\mathrm{G} 94^{50}$ program.

To obtain more accurate energetic information for rate calculations, the potential energy along the MEP was scaled by a factor of 1.123 to reproduce the classical barrier height calculated at the more accurate $\operatorname{CCSD}(\mathrm{T}) / 6-31 \mathrm{G}(\mathrm{d}, \mathrm{p}) / / \mathrm{MP} 2 / 6$ $31 \mathrm{G}(\mathrm{d}, \mathrm{p})$ level of theory. ${ }^{40,51}$ Rate calculations were carried out using our TheRate (theoretical rate $)^{52}$ program.

\section{Analysis of Kinetic Isotope Effects}

A. Contributions to the Kinetic Isotope Effect. The monodeuterated kinetic isotope effect in this study is defined by

$$
\mathrm{KIE}(T) \equiv \frac{k_{\mathrm{HH}}^{\mathrm{CVT} / \mathrm{SCT}}(T)}{k_{\mathrm{HD}}^{\mathrm{CVT} / \mathrm{SCT}}(T)}
$$

where the subscripts $\mathrm{HH}$ and HD denote rate constants for the protonated and monodeuterated systems, respectively. This can be decomposed into individual factors from tunneling $(\kappa)$, rotational (rot), and vibrational (vib) motions, and also from the potential energy $(V)$ due to the isotopic shift of the variational transition state, and is given by

$$
\mathrm{KIE}(T)=\eta_{\kappa} \eta_{\mathrm{rot}}^{\mathrm{CVT}} \eta_{\mathrm{vib}}^{\mathrm{CVT}} \eta_{V}^{\mathrm{CVT}}
$$

where the $\eta_{\mathrm{rot}}^{\mathrm{CVT}}$ and $\eta_{\mathrm{vib}}^{\mathrm{CVT}}$ factors are defined as

$$
\eta_{\mathrm{rot}}^{\mathrm{CVT}}=\frac{Q_{\mathrm{rot}, \mathrm{HH}}^{\mathrm{GT}}\left(T, \mathrm{~s}_{*}{ }^{\mathrm{CVT}}\right) Q_{\mathrm{rot}, \mathrm{HD}}^{\mathrm{R}}(T)}{Q_{\mathrm{rot}, \mathrm{HD}}^{\mathrm{GT}}\left(T, \mathrm{~s}_{*}{ }^{\mathrm{CVT}}\right) Q_{\mathrm{rot}, \mathrm{HH}}^{\mathrm{R}}(T)}
$$


and

$$
\eta_{\mathrm{vib}}^{\mathrm{CVT}}=\frac{Q_{\mathrm{vib}, \mathrm{HH}}^{\mathrm{GT}}\left(T,,_{*}{ }^{\mathrm{CVT}}\right) Q_{\mathrm{vib}, \mathrm{HD}}^{\mathrm{R}}(T)}{Q_{\mathrm{vib}, \mathrm{HD}}^{\mathrm{GT}}\left(T, S_{*}{ }^{\mathrm{CVT}}\right) Q_{\mathrm{vib}, \mathrm{HH}}^{\mathrm{R}}(T)}
$$

where the generalized transition state partition functions are evaluated at the CVT transition state, $s_{*}{ }^{\mathrm{CVT}}$. The effect on the $\mathrm{KIE}$ of the potential is incorporated in

$$
\eta_{V}^{\mathrm{CVT}}=\exp \left[-\beta\left(V_{\mathrm{MEP}, \mathrm{HH}}\left(s_{*}{ }^{\mathrm{CVT}}\right)-V_{\mathrm{MEP}, \mathrm{HD}}\left({ }_{*}{ }^{\mathrm{CVT}}\right)\right)\right]
$$

where each $V_{\mathrm{MEP}}\left(s_{*}{ }^{\mathrm{CVT}}\right)$ is the classical potential energy calculated at the corresponding CVT transition state. The tunneling factor is given by the ratio of the transmission coefficients

$$
\eta_{\kappa}=\frac{\kappa_{\mathrm{HH}}^{\mathrm{SCT}}(T)}{\kappa_{\mathrm{HD}}^{\mathrm{SCT}}(T)}
$$

In our previous study, ${ }^{40}$ we found that the $\mathrm{OH}$ and ND modes are strongly coupled to the reaction coordinate. For this reason, we expect these two modes would also have large contributions to the KIE. For analysis purposes, we further decomposed the vibrational contribution to the KIE, $\eta_{\text {vib }}^{\mathrm{CVT}}$, into contributions from the two active $\mathrm{OH}$ and $\mathrm{NH}$ modes, other stretches and bends as given by

$$
\eta_{\text {vib }}^{\mathrm{CVT}}=\eta_{\mathrm{OH}}^{\mathrm{CVT}} \eta_{\mathrm{NH}}^{\mathrm{CVT}} \eta_{\text {stretches }}^{\mathrm{CVT}} \eta_{\text {bends }}^{\mathrm{CVT}}
$$

where each $\eta_{x}^{\mathrm{CVT}}$ is defined as

$$
\eta_{x}^{\mathrm{CVT}}=\frac{\mathrm{q}_{x, \mathrm{HH}}^{\mathrm{CVT}}}{\mathrm{q}_{x, \mathrm{HD}}^{\mathrm{CVT}}} \frac{\mathrm{q}_{x, \mathrm{HD}}^{\mathrm{R}}}{\mathrm{q}_{x, \mathrm{HH}}^{\mathrm{R}}} \quad(x=\mathrm{OH} \text { and NH mode })
$$

and

$$
\eta_{y}^{\mathrm{CVT}}=\prod_{y} \frac{\mathrm{q}_{y, \mathrm{HH}}^{\mathrm{CVT}} \mathrm{q}_{y, \mathrm{HD}}^{\mathrm{R}}}{\mathrm{q}_{y, \mathrm{HD}}^{\mathrm{CVT}} \mathrm{q}_{y, \mathrm{HH}}^{\mathrm{R}}} \quad(y=\text { bending and stretching modes })
$$

Stretches are defined as modes with frequencies greater than $1800 \mathrm{~cm}^{-1}$, and bends are defined as modes with frequencies less than $1800 \mathrm{~cm}^{-1}$ in the reactants and products. Vibrational modes of the transition state classified as bends or stretches are based on the frequencies to which they correlate in the reactant and product channels (see Figures 2 and 3).

B. Rule of the Geometric Mean. The rule of the geometric mean

$$
\frac{k_{\mathrm{HH}}}{k_{\mathrm{HD}}}=\frac{k_{\mathrm{HD}}}{k_{\mathrm{DD}}}
$$

rests on the following assumptions: (i) rotational partition functions are assumed to cancel and the vibrational partition functions within the harmonic approximation are evaluated in the high-temperature limit, (ii) there is no tunneling along the reaction coordinate, (iii) no secondary kinetic isotope effects and (iv) reactive sites are chemically equivalent (chemical equivalence allows for the cancellation of the vibrational partition functions).

Here, we examine the applicability of this rule to the concerted double proton transfer of this study by calculating the three rate constants $k_{\mathrm{HH}}, k_{\mathrm{HD}}$, and $k_{\mathrm{DD}}$ for the temperature range $200-1000 \mathrm{~K}$. Though the validity of eq 14 requires

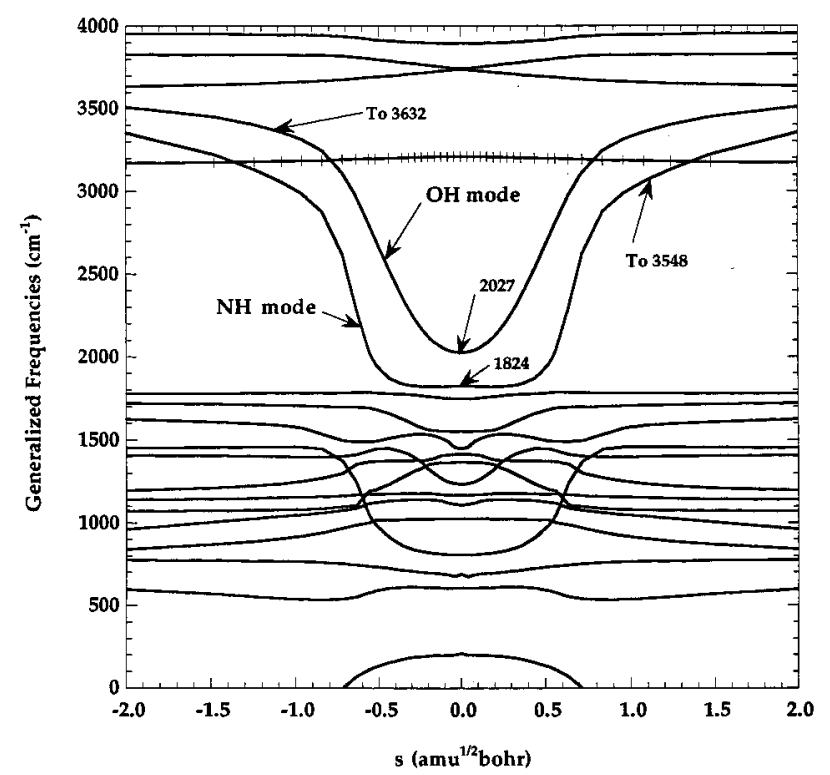

Figure 2. Plot of generalized frequencies vs reaction coordinate for the double-proton system. Important modes and frequencies are highlighted. Bars indicate where Hessians are available. Some low frequencies were not shown.

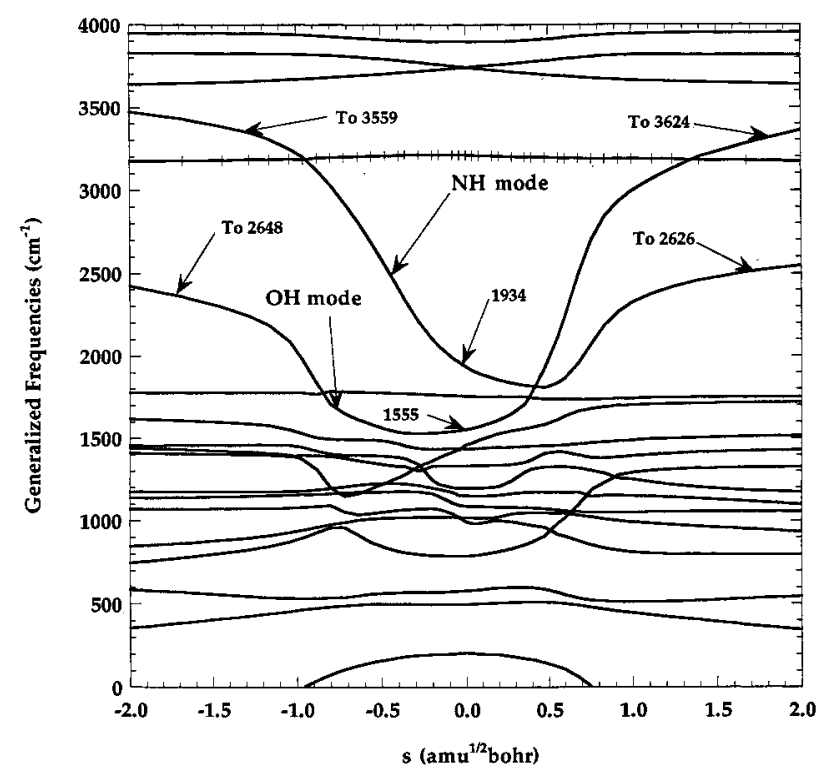

Figure 3. Similar to Figure 2 except for the proton-deuterium system.

chemically equivalent sites, we carry out this calculation by making the assumption that the $\mathrm{NH}$ and $\mathrm{OH}$ bonds of the formamidine-water complex are nearly chemically equivalent. This assumption is based upon the fact that the difference in the forward and reverse zero-point corrected barriers for the monodeuterated system (see Figure 1) is only $0.04 \mathrm{kcal} / \mathrm{mol}$. More importantly, we believe that the quantal effects in the vibrational motions and tunneling through the potential barrier are the important factors for deviation from the rule of the geometric mean. This expectation is in fact confirmed in this study.

\section{Results and Discussion}

As we reported in a previous study ${ }^{40}$ of this system, singlepoint energy calculations at the $\operatorname{CCSD}(\mathrm{T}) / 6-31 \mathrm{G}(\mathrm{d}, \mathrm{p})$ level at the MP2/6-31G(d,p) optimized geometries yielded classical and zero-point energy barriers of 21.9 and $17.8 \mathrm{kcal} / \mathrm{mol}$, respectively, for the double-protonated system. On the basis of our previous studies, ${ }^{40,51}$ the level of theory utilized is accurate enough for the purposes of this study. 


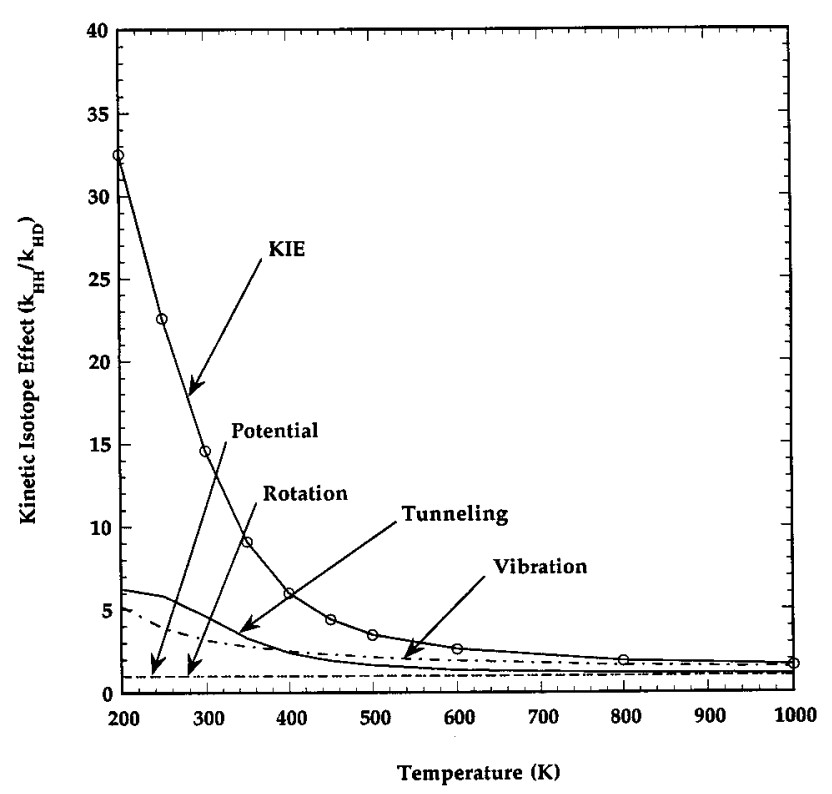

Figure 4. Calculated CVT/SCT solvent kinetic isotope effects and their individual components for the double proton-transfer reaction in the formamidine-water complex (forward reaction). Circles indicate where data is available.

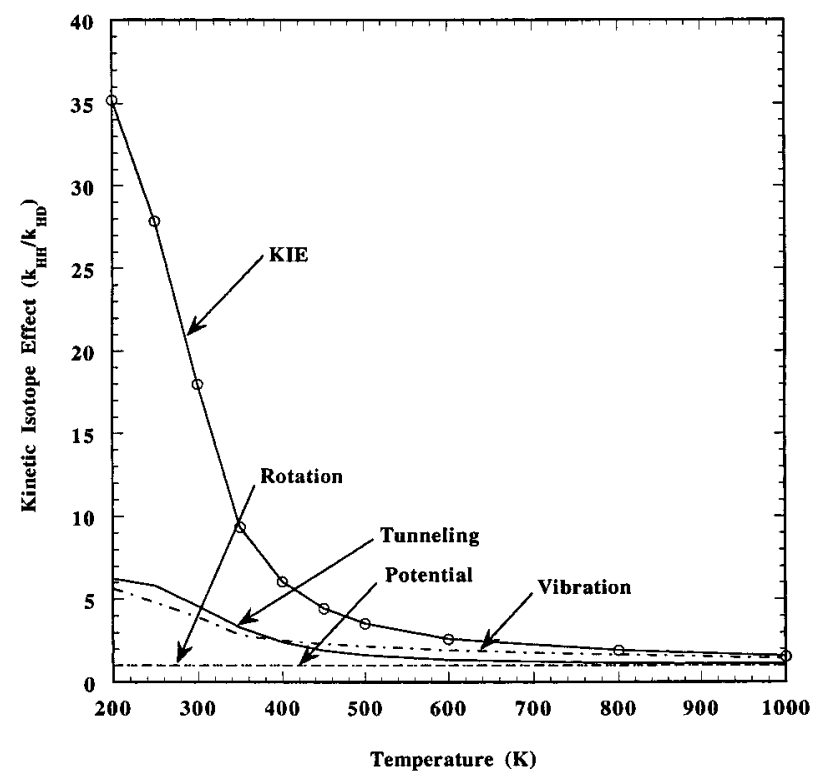

Figure 5. Similar to Figure 4 except for the primary kinetic isotope effect (reverse reaction).

In this study, we find that for the monodeuterated system, the forward zero-point energy barrier is $16.08 \mathrm{kcal} / \mathrm{mol}$ while for the reverse it is $16.12 \mathrm{kcal} / \mathrm{mol}$. The zero-point energy barrier of the double-deuterated system is $16.81 \mathrm{kcal} / \mathrm{mol}$.

A. Total Kinetic Isotope Effect. The solvent (forward reaction) and primary (reverse reaction) KIEs display similar features (see Figures 4 and 5). As expected, the magnitude of the KIEs and the components decrease as the temperature increases for both KIEs. The magnitude of the total solvent KIE decreases from 32.5 to 1.61 as the temperature increases from 200 to $1000 \mathrm{~K}$. The magnitude of the total primary (reverse reaction) KIE shows similar behavior, decreasing from 35.2 to 1.56 with increasing temperature from 200 to $1000 \mathrm{~K}$. The primary KIEs are larger than that of the solvent, particularly at low temperatures, for instance by $23 \%$ at $300 \mathrm{~K}$.

B. Individual Components of the Total KIE. 1. The Potential and Rotational Component. In Figures 4 and 5, the contibution from the potential factor, $\eta_{V}$, is close to 1.00 over

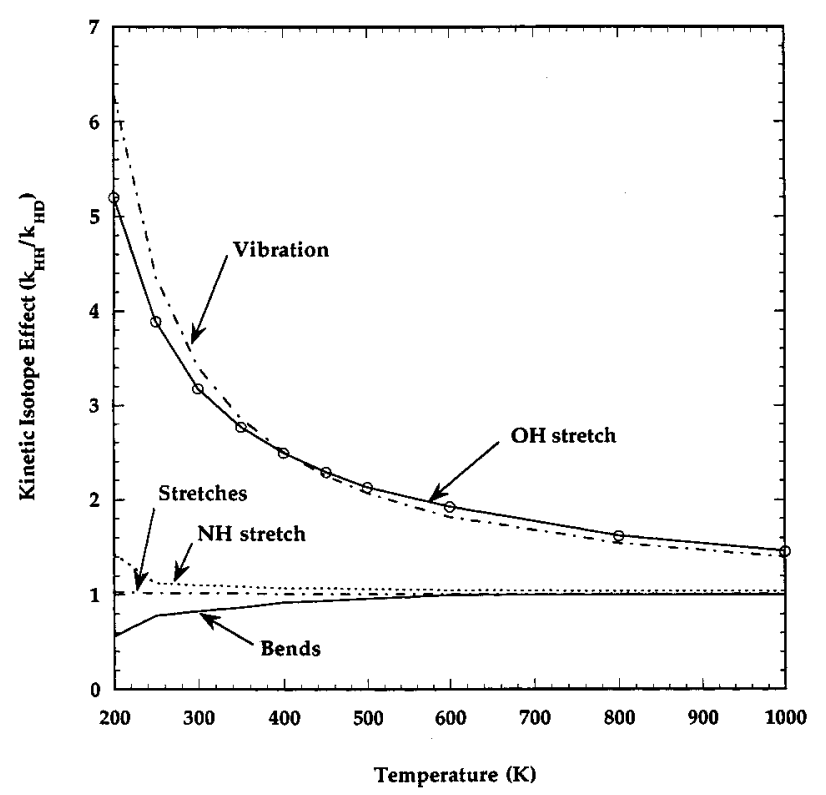

Figure 6. Individual contributions to the vibrational components of the solvent kinetic isotope effect for the double proton-transfer reaction in the monodeuterated formamidine-water complex forward reaction. Circles indicate where data is available.

the entire temperature range. This is due to a negligible shift in the CVT dynamical bottleneck upon deuteration. For instance, the CVT transition state lies at $-0.007 \mathrm{amu}^{1 / 2} \mathrm{a}_{0}$ at $300 \mathrm{~K}$. The rotational factor shows a similar behavior, also being about 1.00 , for the temperature range from 200 to 1000 $\mathrm{K}$. This result is expected since changes in the moments of inertia of the structure are negligible upon deuteration due to the small mass of a hydrogen atom compared to those of the $\mathrm{C}$, $\mathrm{N}$, and $\mathrm{O}$ atoms.

2. Tunneling and Vibrational Components. The tunneling and vibrational components of the total KIE are also shown in Figures 4 and 5. Note that the tunneling component $\eta_{\kappa}$ has the same magnitude in both the solvent and primary KIEs. As expected, the magnitude of the tunneling contribution increases as the temperature decreases, particularly from 1.0 to 6.25 as the temperature drops from 1000 to $200 \mathrm{~K}$. However, it is surprising to find that the vibrational components for both solvent and primary KIEs also show similar magnitudes. In particular, for the solvent KIE, the vibrational component decreases monotonically from 5.20 to 1.46 as the temperature drops from 1000 to $200 \mathrm{~K}$. For the primary KIE, this component varies from 5.64 at $200 \mathrm{~K}$ to 1.42 at $1000 \mathrm{~K}$.

The larger zero-point energy corrected barrier for the reverse reaction of $0.04 \mathrm{kcal} / \mathrm{mol}$ also contributed in a $\eta_{\text {vib }}$ component for the primary KIE being larger than that for the solvent by $19 \%$ at $300 \mathrm{~K}$.

At low temperatures, particularly below $400 \mathrm{~K}$, the tunneling components for both the solvent and primary KIE were found to be larger than the vibrational ones. For instance, at $300 \mathrm{~K}$, the tunneling component is larger by $44 \%$ in the solvent KIE and $17 \%$ in the primary KIE.

The surprisingly large vibrational component in both the primary and solvent KIEs motivated us to further investigate the nature of vibrational modes that are responsible for such effects. To do this, we factored the total KIE into individual contributions from bends, inactive stretches, and the active $\mathrm{NH}$ and $\mathrm{OH}$ stretches as described by eq 12 . The vibrational component of the solvent and primary KIEs displayed in Figures 6 and 7 show interesting similarities and differences. In both cases, it was found that certain modes show normal while others 


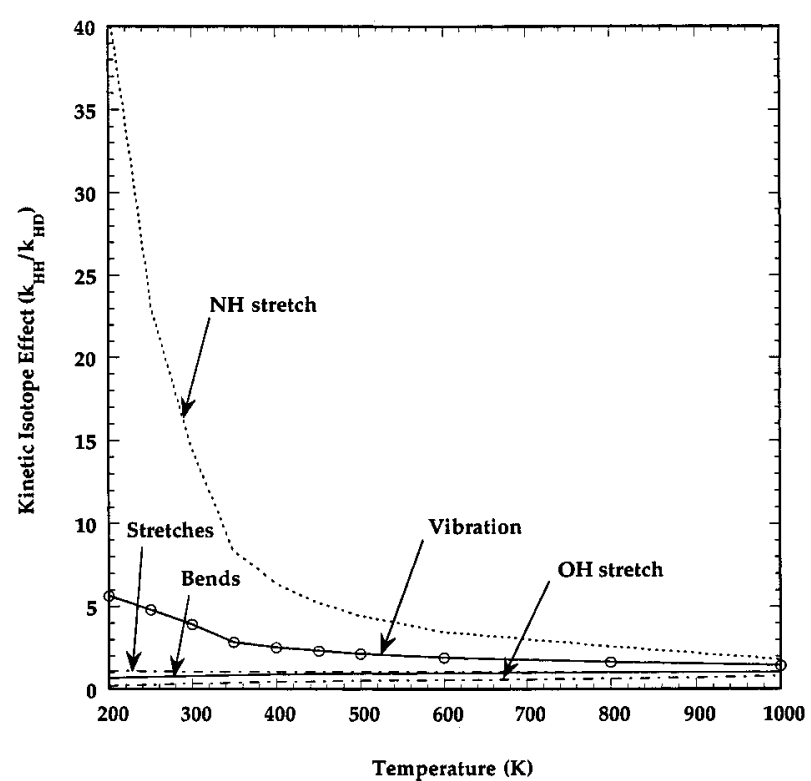

Figure 7. Similar to Figure 6 except for the primary kinetic isotope effect (reverse reaction).

yield inverse KIEs. The normal KIE can be explained as follows. Deuteration yields a decrease in the zero-point energy in the reactant greater than in the transition state (see Figure 8a). The net effect of deuteration is an increase in the zeropoint energy corrected barrier. The opposite effect, i.e., an inverse KIE, can be seen in Figure 8b. Here deuteration lowers the zero-point energy in the transition state more than in the reactant, which results in a decrease in the zero-point energy corrected barrier.

The contributions from stretches, $\eta_{\text {stretches }}$, to $\eta_{\text {vib }}$ for both KIEs are small and normal, which indicates that they behave as spectator modes and are sufficiently decoupled from the reaction coordinate. Over the entire temperature range studied here, they vary from 1.03 to 1.01 .

The contributions from bending modes, $\eta_{\text {bends }}$, show significant inverse KIEs for both reactions (see Figures 6 and 7). They vary from 0.56 for the solvent KIE and from 0.67 for the primary KIE to about 1.00 as the temperature is increased from 200 to $1000 \mathrm{~K}$. An examination of the effect of deuteration on the reactant, product, and transition state bending modes shows that all decrease in frequency (see Table 1). The decrease in the zero-point energy of the bending modes is greater in the transition state than it is in the reactant and product structures. Thus, the bending modes effectively lower the zero-point corrected energy barriers for both reactions and hence yield inverse KIEs (see Figure 8b). In particular, the decrease in total zero-point energy at the transition state upon deuteration due to the bending modes is $1.04 \mathrm{kcal} / \mathrm{mol}$, while it is 0.79 at the reactant and $0.86 \mathrm{kcal} / \mathrm{mol}$ at the product (see Table 2). Thus these bending modes upon deuteration lead to decreases in the zero-point energy corrected barriers by 0.25 and $0.18 \mathrm{kcal} / \mathrm{mol}$, respectively, for the solvent and primary KIEs.

As for the contributions from the $\mathrm{NH}$ and $\mathrm{OH}$ modes, differences in each reaction can be seen in Figures 6 and 7. For the solvent $\mathrm{KIE}$, the $\mathrm{OH}$ contribution is normal $(\geq 1)$ and the $\mathrm{NH}$ contribution is also normal but small. However, for the primary KIE, the $\mathrm{NH}$ contribution is normal but large while the $\mathrm{OH}$ mode exhibits a large inverse $(<1)$ contribution. For instance, at $300 \mathrm{~K}$, contribution factors to the solvent $\mathrm{KIE}$ are 3.41 for the $\mathrm{OH}$ mode and 1.10 for the $\mathrm{NH}$ mode, while these factors in the primary KIE are 0.33 for the $\mathrm{OH}$ and 14.5 for the NH modes. a)
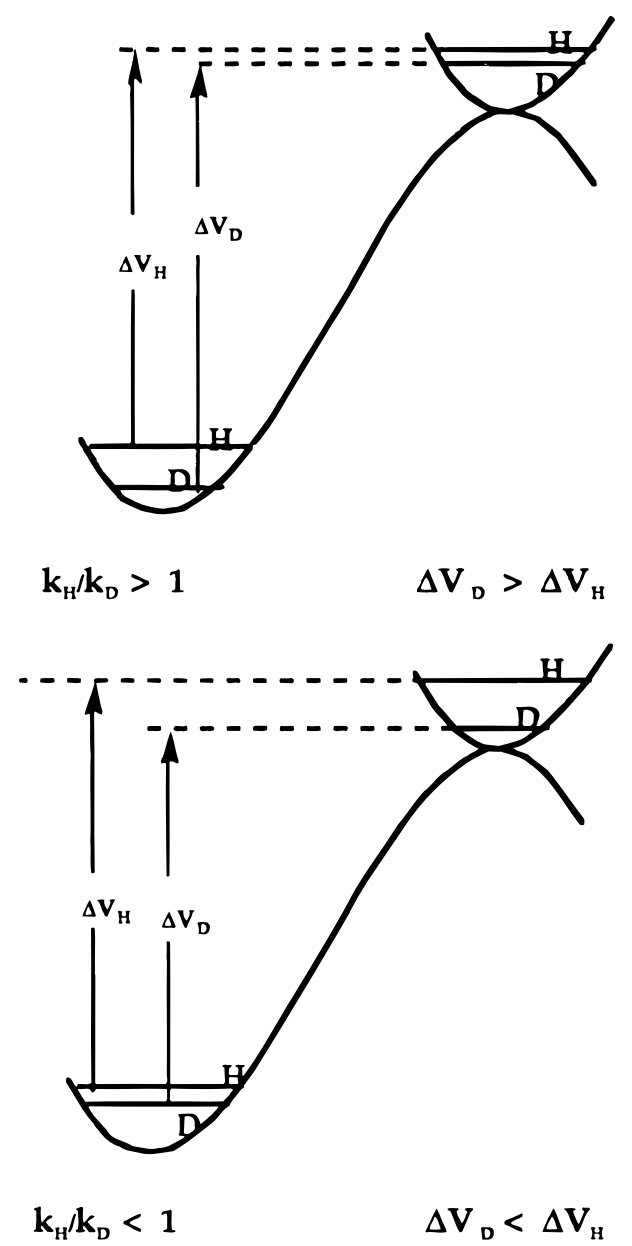

Figure 8. Schematic on the relationship between zero-point energy corrected barriers and kinetic isotope effects. (a) The barrier of the deuterated system is larger resulting in a kinetic isotope effect greater than 1. (b) The barrier of the deuterated system is smaller resulting in a kinetic isotope effect less than one.

The contributions of the $\mathrm{OH}$ and $\mathrm{NH}$ modes to the KIE are consistent with their changes in vibrational frequencies and thus their changes in zero-point energy corrected barriers upon deuteration as shown in Table 3. For instance, the zero-point energy of the $\mathrm{OH}$ mode in the reactant decreased by $1.41 \mathrm{kcal} /$ mol upon deuteration. In the transition state, it decreased by $0.68 \mathrm{kcal} / \mathrm{mol}$. As a result, it contributes to an increase in the zero-point energy corrected barrier by $0.73 \mathrm{kcal} / \mathrm{mol}$. This leads to a normal contribution to the solvent KIE from the $\mathrm{OH}$ mode. This situation is reversed for the primary KIE. The zero-point energy of the $\mathrm{OH}$ mode in the product decreased by only 0.01 $\mathrm{kcal} / \mathrm{mol}$. Effectively, it contributes to a decrease in the zeropoint energy corrected barrier of $0.67 \mathrm{kcal} / \mathrm{mol}$ and therefore leads to an inverse KIE contribution.

As for the $\mathrm{NH}$ mode, it displays increases in its zero-point energy in both the reactant and transition state by 0.02 and 0.15 $\mathrm{kcal} / \mathrm{mol}$, respectively, while in the product it decreases by 1.32 $\mathrm{kcal} / \mathrm{mol}$. Consequently, these changes contribute to increases in the zero-point energy corrected barriers by 0.13 and 1.47 $\mathrm{kcal} / \mathrm{mol}$ for the solvent and primary KIEs, respectively, and thus yields normal contributions.

A comparison of the various levels of rate calculation is in order. The solvent KIEs for the TST, CVT, TST/W and CVT/ SCT levels are 3.17, 3.18, 3.91, and 17.5, respectively. There is a small difference between the TST and CVT levels. Of particular note is the large difference between the TST/W and CVT/SCT levels. The CVT/SCT value is larger than the TST/W value by a factor of 4.5 , which is significant, especially in the 
TABLE 1: Reactant, Transition State, and Product Vibrational Frequencies $\left(\mathrm{cm}^{-1}\right)$ in the Harmonic Approximation for the Protonated and Monodeuterated Formamidine-Water Complex

\begin{tabular}{|c|c|c|c|c|c|}
\hline \multicolumn{2}{|c|}{ reactant } & \multicolumn{2}{|c|}{ transition state } & \multicolumn{2}{|c|}{ product } \\
\hline protonated & deuterated & protonated & deuterated & protonated & deuterated \\
\hline \multicolumn{6}{|c|}{ Bends } \\
\hline 155 & 155 & $1643 i$ & $1414 i$ & 155 & 149 \\
\hline 180 & 179 & 209 & 209 & 180 & 178 \\
\hline 221 & 218 & 436 & 434 & 221 & 218 \\
\hline 296 & 294 & 484 & 473 & 296 & 295 \\
\hline 376 & 321 & 501 & 499 & 376 & 372 \\
\hline 430 & 429 & 598 & 580 & 430 & 425 \\
\hline 591 & 578 & 607 & 604 & 591 & 549 \\
\hline 763 & 615 & 689 & 688 & 763 & 609 \\
\hline 821 & 763 & 807 & 789 & 821 & 810 \\
\hline 826 & 823 & 1026 & 995 & 826 & 817 \\
\hline 1067 & 1066 & 1109 & 1020 & 1067 & 1054 \\
\hline 1143 & 1143 & 1168 & 1095 & 1143 & 1073 \\
\hline 1179 & 1172 & 1236 & 1160 & 1179 & 1152 \\
\hline 1400 & 1400 & 1368 & 1197 & 1400 & 1318 \\
\hline 1452 & 1452 & 1448 & 1333 & 1452 & 1436 \\
\hline 1667 & 1466 & 1475 & 1436 & 1667 & 1538 \\
\hline 1722 & 1666 & 1554 & 1462 & 1722 & 1721 \\
\hline 1778 & 1778 & 1747 & 1757 & 1778 & 1753 \\
\hline \multicolumn{6}{|c|}{ Stretches $^{a}$} \\
\hline$(\mathrm{CH}) 3167$ & 3167 & 3209 & 3209 & 3167 & 3167 \\
\hline$(\mathrm{NH}) 3548^{b}$ & 3559 & 1824 & 1934 & 3548 & 2626 \\
\hline$(\mathrm{NH}) 3618$ & 3618 & 3739 & 3739 & 3618 & 3616 \\
\hline$(\mathrm{OH}) 3632^{b}$ & 2648 & 2027 & 1555 & 3632 & 3623 \\
\hline$(\mathrm{NH}) 3792$ & 3792 & 3743 & 3743 & 3792 & 3772 \\
\hline$(\mathrm{OH}) 3958$ & 3948 & 3897 & 3897 & 3958 & 3958 \\
\hline
\end{tabular}

${ }^{a}$ Stretches are correlated from reactants to products whereas bends are not. ${ }^{b}$ Bonds formed and broken in reaction.

TABLE 2: Changes in Total Zero-Point Energies (kcal/mol) of Vibrational Modes of Reactant, Product, and Transition State Upon Deuteration and Shifts in the Zero-Point Energy Corrected Barriers $\Delta V_{\mathrm{a}, \mathrm{f}}^{\mathrm{G}}$ and $\Delta V_{\mathrm{a}, \mathrm{r}}^{\mathrm{G}}(\mathrm{kcal} / \mathrm{mol})$ for the Forward and Reverse Reactions, Respectively, in the Formamidine-Water Complex

\begin{tabular}{lccccc}
\hline & \multicolumn{5}{c}{ total zero-point energies of } \\
vibrational \\
\cline { 2 - 4 } modes $^{*}$ & reactant & transition state & product & $\Delta V_{\mathrm{a}, \mathrm{f}}^{\mathrm{G}}$ & $\Delta V_{\mathrm{a}, \mathrm{r}}^{\mathrm{G}}$ \\
\hline bends(HH) & 22.97 & 23.53 & 22.97 & & \\
bends(HD) & 22.18 & 22.49 & 22.11 & & \\
$\Delta(\mathrm{HH} \rightarrow \mathrm{HD})$ & -0.79 & -1.04 & -0.86 & -0.25 & -0.18 \\
stretches(HH) & 20.78 & 20.58 & 20.78 & & \\
stretches(HD) & 20.76 & 20.85 & 20.75 & & \\
$\Delta(\mathrm{HH} \rightarrow \mathrm{HD})$ & -0.02 & +0.27 & -0.03 & +0.29 & +0.30
\end{tabular}

${ }^{a}$ Modes (HH) and modes (HD) refer to a property of the modes in the protonated and monodeuterated molecules, respectively. See Table 1 for the classification of bends and stretches. $\Delta \mathrm{H} \rightarrow \mathrm{D}$ refers to the change in property upon single deuteration.

context of this work. This indicates that the Wigner level for tunneling correction is inadequate for the purposes of this study.

3. Rule of the Geometric Mean. Figure 9 shows the deviations (i.e. deviations from unity) from the RGM or classical behavior of the various rate constants for the solvent KIE. The two rate constants where no tunneling is included, namely those of TST and CVT, show significant deviations from classical behavior at low temperatures. This is due to the fact that at low temperatures quantal effects in vibrational partition functions become important and thus the assumptions of classical partition function with small quantal correction is no longer valid. Such assumption is quite good at high temperatures (see Figure 9). The CVT/SCT ratio displays the largest deviation from this rule. This is due to quantum effects in both the vibrational motions and the reaction coordinate (tunneling). However, tunneling is
TABLE 3: Changes in Total Zero-Point Energies (kcal/mol) of Active Vibrational Modes of Reactant, Product and Transition State Upon Deuteration and

Shifts in the Zero-Point Energy Corrected Barriers $\Delta V_{\mathrm{a}, \mathrm{f}}^{\mathrm{G}}$ and $\Delta V_{\mathrm{a}, \mathrm{r}}^{\mathrm{G}}(\mathrm{kcal} / \mathrm{mol})$ for the Forward and Reverse Reactions, Respectively, in the Formamidine-Water Complex

\begin{tabular}{|c|c|c|c|c|c|}
\hline \multirow{2}{*}{$\begin{array}{l}\text { individual } \\
\text { modes }^{a}\end{array}$} & \multicolumn{3}{|c|}{$\begin{array}{l}\text { zero-point energies of individual } \\
\text { active vibrational modes }\end{array}$} & \multirow[b]{2}{*}{$\Delta V_{\mathrm{a}, \mathrm{f}}^{\mathrm{G}}$} & \multirow[b]{2}{*}{$\Delta V_{\mathrm{a}, \mathrm{r}}^{\mathrm{G}}$} \\
\hline & reactant & transition state & product & & \\
\hline $\mathrm{OH}(\mathrm{HH})$ & 5.19 & 2.90 & 5.19 & & \\
\hline $\mathrm{OH}(\mathrm{HD})$ & 3.78 & 2.22 & 5.18 & & \\
\hline$\Delta \mathrm{HH} \rightarrow \mathrm{HD}$ & -1.41 & -0.68 & -0.01 & +0.73 & -0.67 \\
\hline $\mathrm{NH}(\mathrm{HH})$ & 5.07 & 2.61 & 5.07 & & \\
\hline $\mathrm{NH}(\mathrm{HD})$ & 5.09 & 2.76 & 3.75 & & \\
\hline$\Delta \mathrm{HH} \rightarrow \mathrm{HD}$ & +0.02 & +0.15 & -1.32 & +0.13 & +1.47 \\
\hline
\end{tabular}

${ }^{a}$ Mode (HH) and mode (HD) refer to a property of the mode in the protonated and monodeuterated molecules, respectively. See Table 5.1 for the classification of bends and stretches. $\Delta \mathrm{HH} \rightarrow \mathrm{HD}$ refers to the change in property upon single deuteration.

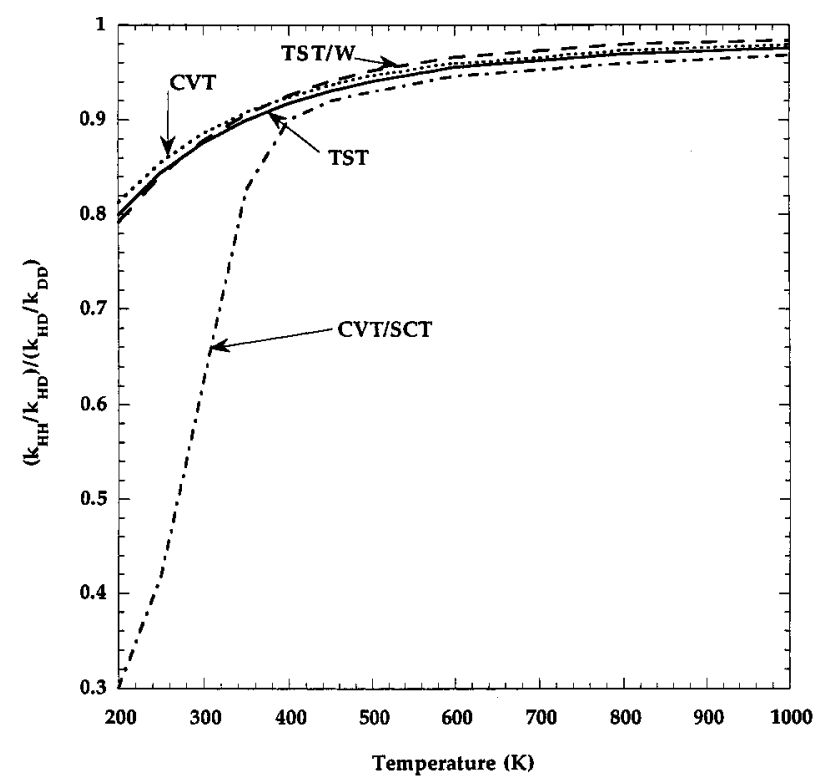

Figure 9. Plot of deviations from the rule of the geometric mean vs temperature (K) for rate constants TST (solid), TST/W (dash), CVT (dotted), and CVT/SCT (dash dotted).

a far more important factor for this deuteration particularly at low temperatures.

\section{Conclusion}

In this study, using an ab initio direct dynamics methodology, we have examined in detail the primary and solvent KIE of the water-assisted tautomerization of the formamidine-water complex. In particular, we found that the primary and solvent KIEs are large at low temperatures. However, it is not only due to tunneling effects as one normally expected but also to the quantum effects in the vibrational motions. In fact, both contributions have similar magnitudes. For tunneling contributions, the Wigner correction significantly underestimates the tunneling probability and consequently predicts the KIE to be too small at low temperatures. By examining individual contibutions of different vibrational modes we are able to understand the difference in the total primary and solvent KIEs, and how the two active $\mathrm{OH}$ and $\mathrm{NH}$ stretches can have drastic different contributions to the overall KIE.

We have used this reaction as a means to examine the validity of the rule of the geometric mean for concerted double proton- 
transfer reactions. We found that at low temperatures (below $400 \mathrm{~K}$ in this case) quantum effects in the vibrational motions and tunneling become important. This leads to a significant deviation from the rule of the geometric mean, which assumes no tunneling along the reaction coordinate and small quantal effects in the vibrational motions.

\section{References and Notes}

(1) Swain, C. G.; Brown, J. F. J. Am. Chem. Soc. 1952, 74, 2534; Ibid. 1952, 74, 2538.

(2) Gandour, R. D.; Schowen, R. L. Transition State of Biochemical Processes; Plenum Press: New York, 1978.

(3) Engdahl, K. A.; Bivehed, H.; Ahlberg, P.; Saunders, W. H., Jr. J. Chem. Soc., Chem. Commun. 1982, 423.

(4) Hermes, J. D.; Morrical, S. W.; O’Leary, M. H.; Cleland, W. W. Biochemistry 1984, 23, 5479.

(5) Belasco, J. G.; Albery, W. J.; Knowles, J. R. Biochemistry 1986, $25,2552$.

(6) Wong, K. K.; Vanoni, M. A.; Blanchard, J. S. Biochemistry 1988, 27, 7091.

(7) Duggleby, R. G.; Northrop, D. B. Bioorg. Chem. 1989, 17, 177.

(8) Denu, J. M.; Fitzpatrick, P. F. Biochemistry 1994, 33, 4001.

(9) Scherer, G.; Limbach, H-H. J. Am. Chem. Soc. 1989, 111, 5946.

(10) Rumpel, H.; Limbach, H-H. J. Am. Chem. Soc. 1989, 111, 5429.

(11) Schlabach, M.; Scherer, G.; Limbach, H-H. J. Am. Chem. Soc. 1991, 113, 3550 .

(12) Hanzlik, R. P.; Schaefer, A. R.; Moon, J. B.; Judson, C. M. J. Am. Chem. Soc. 1987, 109, 4926.

(13) Baldwin, J. E.; Reddy, V. P. J. Am. Chem. Soc. 1987, 109, 8051

(14) Gajewski, J. J.; Peterson, K. B.; Kagel, J. R.; Huang, Y. C. J. J. Am. Chem. Soc. 1989, 111, 9078.

(15) Poirier, R. A.; Wang, Y.; Westaway, K. C. J. Am. Chem. Soc. 1994, 116,2526

(16) Davidson, M. M.; Hillier, I. H. Chem. Phys. Lett. 1994, 225, 293.

(17) Lu, Da-h.; Maurice, D.; Truhlar, D. G. J. Am. Chem. Soc. 1990 112,6206 .

(18) Houk, K. N.; Gustafson, S. M.; Black, K. A. J. Am. Chem. Soc. $1992,114,8565$.

(19) Storer, J. W.; Raimondi, L.; Houk, K. N. J. Am. Chem. Soc. 1994, $116,9675$.

(20) Glad, S. S.; Jensen, F. J. Am. Chem. Soc. 1994, 116, 9302.

(21) Bach, R. D.; Gonzalez, C.; Andres, J. L.; Schlegel, H. B. J. Org. Chem. 1995, 60, 4653.

(22) Baldwin, J. E.; Reddy, P. J. Am. Chem. Soc. 1988, 110, 8223.

(23) O’Hair, R. A. J.; Davico, G. E.; Hacaloglu, J.; Dang, T. T.; DePuy, C. H.; Bierbaum, V. M. J. Am. Chem. Soc. 1994, 116, 3609.

(24) Garrett, B. C.; Truhlar, D. G.; Bowman, J. M.; Wagner, A. F.; Robie, D.; Arepalli, S.; Presser, N.; Gordon, R. J. J. Am. Chem. Soc. 1986, $108,3515$.

(25) Garrett, B. C.; Truhlar, D. G.; Schatz, G. C. J. Am. Chem. Soc. 1986, $108,2876$.
(26) Fujisaki, N.; Ruf, A.; Gäumann, T. J. Phys. Chem. 1987, 91, 1602. (27) Viggiano, A. A.; Paschkewitz, J. S.; Morris, R. A.; Paulson, J. F.; Gonzalez-Lafont, A.; Truhlar, D. G. J. Am. Chem. Soc. 1991, 113, 9404.

(28) Zhao, X. G.; Tucker, S. C.; Truhlar, D. G. J. Am. Chem. Soc. 1991, 113,826 .

(29) Zhao, X. G.; Lu, Da-H.; Liu, Yi-P.; Lynch, G. C.; Truhlar, D. G. J. Chem. Phys. 1992, 97, 6376.

(30) Liu, Yi-P.; Lu, Da-H.; Gonzalez-Lafont, A.; Truhlar, D. G.; Garrett, B. C. J. Am. Chem. Soc. 1993, 115, 7806 .

(31) Truong, T. N.; Evans, T. J. J. Phys. Chem. 1994, 98, 9558.

(32) Hu, W.-P.; Truhlar, D. G. J. Am. Chem. Soc. 1995, 117, 10726.

(33) Lynch, G. C.; Trular, D. G.; Brown, F. B.; Zhao, J.-g. J. Phys. Chem. 1995, 99, 207.

(34) Gawlita, E.; Szylhabel-Godala, A.; Paneth, P. J. Phys. Org. Chem. 1996, 9, 41.

(35) Bigeleisen, J. J. Chem. Phys. 1955, 23, 2264.

(36) Gold, V. Trans. Faraday Soc. 1960, 56, 255.

(37) Albery, W. J.; Limbach, H. H. Faraday Discuss. Chem. Soc. 1982, $24,291$.

(38) Hermes, J. D.; Cleland, W. W. J. Am. Chem. Soc. 1984, 106, 7263.

(39) Albery, W. J. Phys. Chem. 1986, 90, 3733.

(40) Bell, R. L.; Truong, T. N. J. Chem. Phys. 1994, 101, 10442.

(41) Bell, R. L.; Taveras, D. L.; Truong, T. N.; Simons, J. Int. J. Quantum Chem., in press.

(42) Borman, S. Chem. Eng. News 1994, 72, 25.

(43) Marcus, R. A.; Coltrin, M. E. J. Chem. Phys. 1977, 67, 2609.

(44) Bell, R. P. The Tunnel Effect in Chemistry; Chapman \& Hall: London, 1980.

(45) Lu, Da-h.; Truong. T. N.; Melissas, V. S.; Lynch, G. C.; Liu, YiP.; Garrett, B. C.; Steckler, R.; Isaacson, A. D.; Rai, S. N.; Hancock, G. C.; Lauderdale, J. G.; Joseph, T.; Truhlar, D. G. Comput. Phys. Commun. 1992, 71, 235.

(46) Truhlar, D. G.; Isaacson, A. D.; Garrett, B. C. In Theory of Chemical Reaction Dynamics; Baer, M., Ed.; CRC: Boca Raton, FL, 1985; Vol. 4, pp 65-137.

(47) Truhlar, D. G.; Isaacson, A. D.; Skodje, R. T.; Garrett, B. C. J. Phys. Chem. 1982, 86, 2252.

(48) Gonzalez, C.; Schlegel, B. H. J. Phys. Chem. 1990, 94, 5523.

(49) Duncan, W.; Truong, T. N. To be published.

(50) Frisch, M. J.; Trucks, G. W.; Schlegel, H. B.; Gill, P. M. W.; Johnson, B. G.; Robb, M. A.; Cheeseman, J. R.; Keith, T.; Petersson, G. A.; Montgomery, J. A.; Raghavachari, K.; Al-Laham, M. A.; Zakrzewski, V. G.; Ortiz, J. V.; Foresman, J. B.; Cioslowski, J.; Stefanov, B. B.; Nanayakkara, A.; Challacombe, M.; Peng, C. Y.; Ayala, P. Y.; Chen, W.; Wong, M. W.; Andres, J. L.; Replogle, E. S.; Gomperts, R.; Martin, R. L.; Fox, D. J.; Binkley, J. S.; Defrees, D. J.; Baker, J.; Stewart, J. P.; HeadGordon, M.; Gonzalez, C.; Pople, J. A. Gaussian 94, Revision B.1; Gaussian, Inc.: Pittsburgh, PA, 1995.

(51) Zhang, Q.; Bell, R.; Truong, T. N. J. Phys. Chem. 1995, 99, 592.

(52) Truong, T. N.; Duncan, W. TheRate; The University of Utah: Salt Lake City. More information is available at http://www.chem.utah.edu/ mercury/TheRate/TheRate.html. 